\title{
On the Finite Element Tearing and Interconnecting Method for Scattering by Large 3D Inhomogeneous Targets
}

\author{
Ming-Lin Yang and Xin-Qing Sheng \\ Center for Electromagnetic Simulation, School of Information and Electronics, Beijing Institute of Technology, Beijing 100081, China \\ Correspondence should be addressed to Xin-Qing Sheng, xsheng@bit.edu.cn \\ Received 30 May 2011; Revised 10 August 2011; Accepted 27 August 2011 \\ Academic Editor: Ning Yuan
}

Copyright ( $) 2012$ M.-L. Yang and X.-Q. Sheng. This is an open access article distributed under the Creative Commons Attribution License, which permits unrestricted use, distribution, and reproduction in any medium, provided the original work is properly cited.

The finite element tearing and interconnecting method (FETI) is applied to compute scattering by large 3D inhomogeneous targets. Two algorithms of FETI have been implemented for 3D scattering. The performance of these two FETI algorithms has been investigated in detail, particularly for large inhomogeneous targets. Numerical experiments show that the performance of FETI relies on the style of domain decomposition and inhomogeneity, which has not been carefully studied before. A trick for improving convergence of FETI is presented for inhomogeneous targets.

\section{Introduction}

The domain decomposition has been recognized as one of most important methodologies for constructing efficient parallel computing algorithms in recent years. Among various domain decomposition methods of the finite element method (FEM), the finite element tearing and interconnecting (FETI) shows great potential to improve the capability of the finite element method [1-9]. The FETI designed in [3] was applied to the electromagnetic field problems in [4]. The idea of a more efficient version of FETI, named as FETIDPH designed in [5], was applied to solve large-scale phasedarray antenna and photonic crystal problems and developed as FETI-DPEM1 in [6], where a global preconditioner is smartly designed to significantly improve the convergence of the interface solution in FETI. To maintain high-speed convergence of the interface solution for extremely large-scale problems, the Robin-type transmission conditions at the subdomain interfaces were employed to derive the interface equation instead of Dirichlet-to-Neumann boundary condition in FETI-DPEM1 in [7]. This latest algorithm of FETI in CEM is named as FETI-DPEM2, which shows much better performance than FETI-DPEM1, especially for extremely large-scale problems. Recently, the FETI-DPEM2 is extended to scattering problems [2]. However, few researchers give the detailed investigation on the performance of FETI-DPEM2 for scattering by large inhomogeneous targets.

In this paper, the FETI-DPEM1 and FETI-DPEM2 are implemented for 3D scattering by large inhomogeneous targets. The performance of these two FETI algorithms has been investigated in detail, particularly for large highly inhomogeneous targets and different domain decomposition types. An implementation trick of FETI is presented for inhomogeneous targets.

\section{The FETI-DPEM Formulation}

The 3D scattering by a target can be formulated as

$$
\begin{gathered}
\nabla \times\left(\frac{1}{\mu_{r}} \nabla \times E\right)-k_{0} \varepsilon_{r} E=-j k_{0} Z_{0} J_{\mathrm{imp}}, \quad \text { in } \Omega \in R^{3}, \\
\hat{n} \times E=0, \quad \text { on } \partial \Omega_{\mathrm{PEC}}, \\
\hat{n} \times \nabla \times E=0, \quad \text { on } \partial \Omega_{\mathrm{PMC}}, \\
\hat{n} \times \nabla \times E+j k_{0} \hat{n} \times \hat{n} \times E=U, \quad \text { on } \partial \Omega_{\mathrm{ABC}},
\end{gathered}
$$




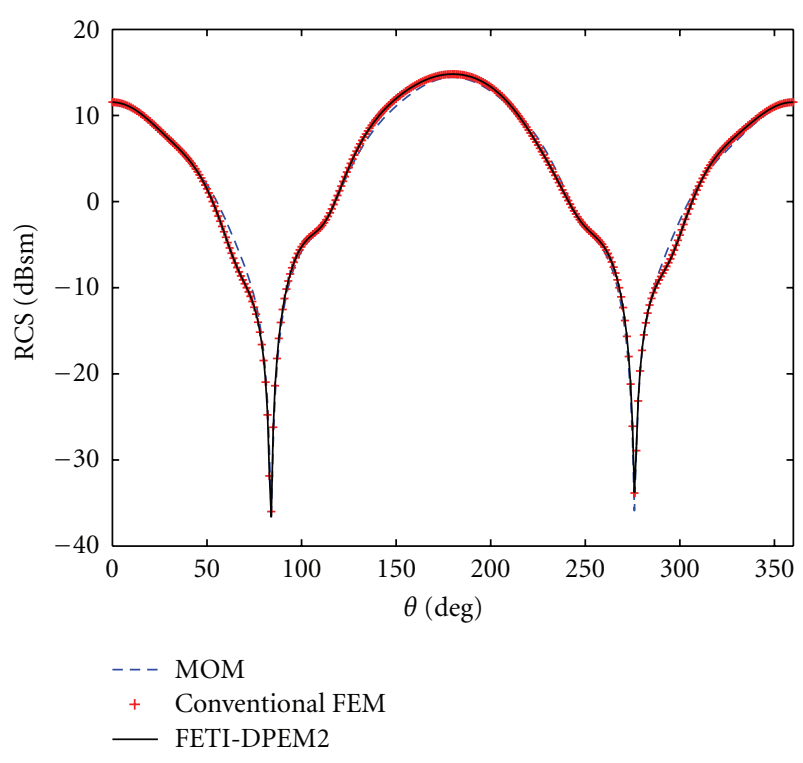

FIGURE 1: Bistatic RCS of the metallic cube with $\theta_{\text {inc }}=0^{\circ}, \varphi_{\text {inc }}=0^{\circ}$ at $0.3 \mathrm{GHz}$.

where $k_{0}$ and $Z_{0}$ are the free-space wave number and intrinsic impedance, respectively, and $J_{\text {imp }}$ is an internal impressed current. $\partial \Omega_{\mathrm{ABC}}$ represents a boundary, where the field satisfies the absorbing boundary condition (ABC) [10] with $U=\hat{n} \times \nabla \times E_{\text {inc }}+j k_{0} \hat{n} \times \hat{n} \times E_{\text {inc }}$ as boundary excitation.

According to the FETI method, the computational domain is divided into nonoverlapping subdomains $\Omega^{p}(p=$ $\left.1,2, \ldots, N_{p}\right)$, where the superscript denotes the subdomain index. The interface shared by the two adjacent subdomain $i$ th and $j$ th is denoted as $\Gamma_{i j}$. The edges shared by more than two subdomains are called corner edges, denoted as $\Gamma_{c}$. The edges shared by two subdomains and the surface of absorbing boundary are also treated as corner edges. The interior fields in each sub-domain still satisfy (1). In the FETI-DPEM1, the boundary condition of each subdomain is formulated by introducing $\Lambda$ as

$$
\hat{n}^{p} \times \frac{1}{\mu_{r}^{p}} \nabla \times E^{p}=-\hat{n}^{q} \times \frac{1}{\mu_{r}^{q}} \nabla \times E^{q}=\Lambda .
$$

In the FETI-DPEM2, the boundary condition of each subdomain is formulated by introducing $\Lambda$ as

$$
\hat{n}^{p} \times \frac{1}{\mu_{r}^{p}} \nabla \times E^{p}+\alpha^{p} \hat{n}^{p} \times \hat{n}^{p} \times E^{p}=\Lambda^{p} .
$$

The fields in each subdomain can be determined under the boundary condition (5) or (6) by using the standard FEM. A special procedure is designed in FETI to solve the fields in each subdomain. The unknowns of the electric field in each subdomain are grouped into three categories

$$
E^{p}=\left[\begin{array}{lll}
E_{V}^{p} & E_{I}^{p} & E_{c}^{p}
\end{array}\right]=\left[\begin{array}{ll}
E_{r}^{p} & E_{c}^{p}
\end{array}\right]
$$

where the subscripts $V, I$, and $c$ denote the unknowns associated with the internal volume, interfaces, and corner edges, respectively. The unknowns $E_{r}^{p}$ associated with the internal volume and interfaces are considered as local variables, whereas the unknowns $E_{c}^{p}$ associated with the corner edges are considered as global variables. The unknowns $E_{r}^{p}$ are eliminated from the FEM matrix equation for the $p$ th subdomain to obtain the corner equation of $E_{c}^{p}$. Afterwards, the obtained corner equations for all sub-domains are assembled together to get the following global corner equation:

$$
\tilde{K}_{c c} E_{c}=\tilde{f}_{c}-K_{r c}^{T} \lambda
$$

where $\tilde{K}_{c c}, \tilde{K}_{r c}^{T}, f_{c}$ can refer to [6]. Solving (8) to obtain $E_{c}$ and then substituting $E_{c}$ back to the FEM matrix equation for each subdomain, we can obtain the expression of $E_{r}^{p}$ in terms of $\lambda$. For FETI-DPEM1, the continuity of tangential electric field between two adjacent subdomain is enforced to $E_{I}^{p}$, and the final equation can be obtained

$$
\left(\widetilde{K}_{r r}+\widetilde{K}_{r c} \tilde{K}_{c c}^{-1} \widetilde{K}_{c r}\right) \lambda=\tilde{f}_{r}-\tilde{K}_{r c} \widetilde{K}_{c c}^{-1} \tilde{f}_{c}
$$

where $\tilde{K}_{r r}, \tilde{K}_{r c}, \tilde{K}_{c r}$, and $\tilde{f}_{r}$ can refer to [6]. For FETI-DPEM2, the continuities of tangential electric and magnetic fields between two adjacent subdomain are enforced to $E_{I}^{p}$, and the final equation has the same form as (9) and has double unknowns compared with FETI-DPEM1.

Since the inverse of $\widetilde{K}_{c c}$ in (9) essentially performs as a global preconditioner, (9) is usually well conditioned. Numerical experiments in $[6,7]$ are performed on a $2 \mathrm{D}$ extended periodical structure radiation problem. Recently, the FETI-DPEM2 is extended to $3 \mathrm{D}$ scattering problems [2]. However, few researchers give the detailed investigation on the performance of FETI for scattering by 3D-extended inhomogeneous targets, especially when the subdomain numbers are increased or material parameters are changed in all $x, y$, and $z$ directions. We will give the detail investigation by numerical experiments in the next section on the performance of FETI for scattering by large inhomogeneous targets.

\section{Numerical Experiments}

To demonstrate the accuracy, efficiency, and capability of the two FETI-DPEM methods, a series of numerical experiments are performed in this section. All the computations are performed on a computer having 2 Intel X5650 $2.66 \mathrm{GHz}$ CPUs with 6 cores for each CPU, 32 GB memory. The GMRES solver in [11] is employed to solve (9) with a restart number of 20, and the convergence criterion is set to 0.005 .

The first numerical experiment is to show the accuracy of the two FETI-DPEM methods. We compute the bistatic RCS of a $1 \mathrm{~m} \times 1 \mathrm{~m} \times 1 \mathrm{~m}$ metallic cube at $0.3 \mathrm{GHz}$ with $\theta_{\text {inc }}=0^{\circ}, \varphi_{\text {inc }}=0^{\circ}$. A tetrahedral mesh with average edge length of $0.05 \mathrm{~m}$ is used. In this calculation, the total FEM computation domain is terminated by enclosing the cube with a cubic outer boundary placed $0.5 \mathrm{~m}$ from the cube. Numerical results obtained are compared in Figure 1 with those obtained by the conventional FEM, and the MOMbased solution using the commercial software FEKO. Good agreement can be observed among these results. 




(a) 2D extended

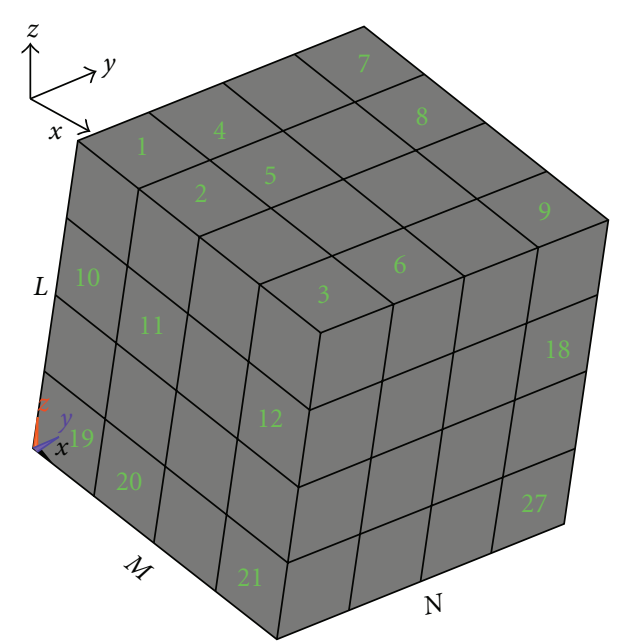

(b) 3D extended

FIgURE 2: Domain decomposition style of a brick domain.

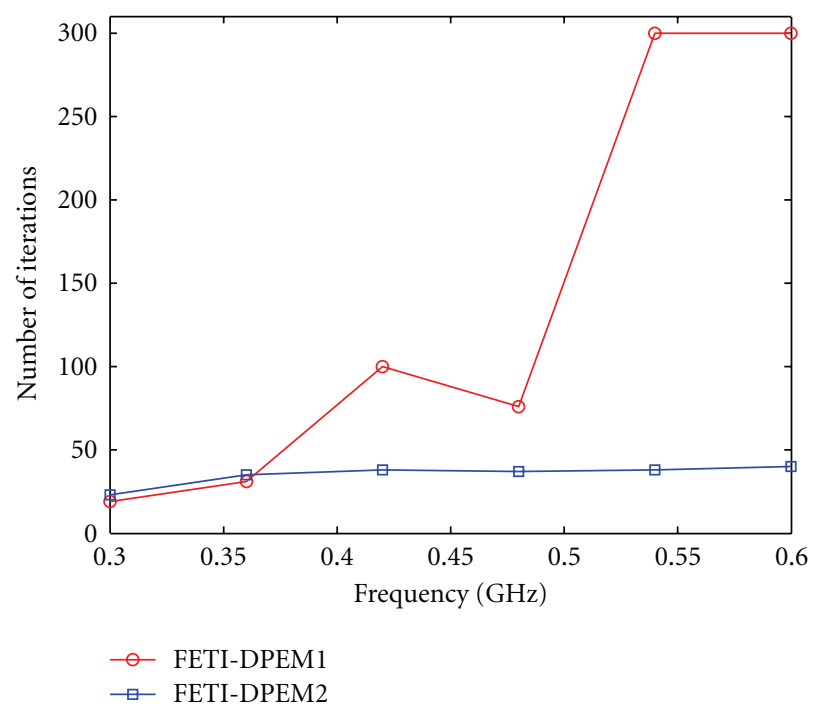

(a) Iteration number versus frequency.

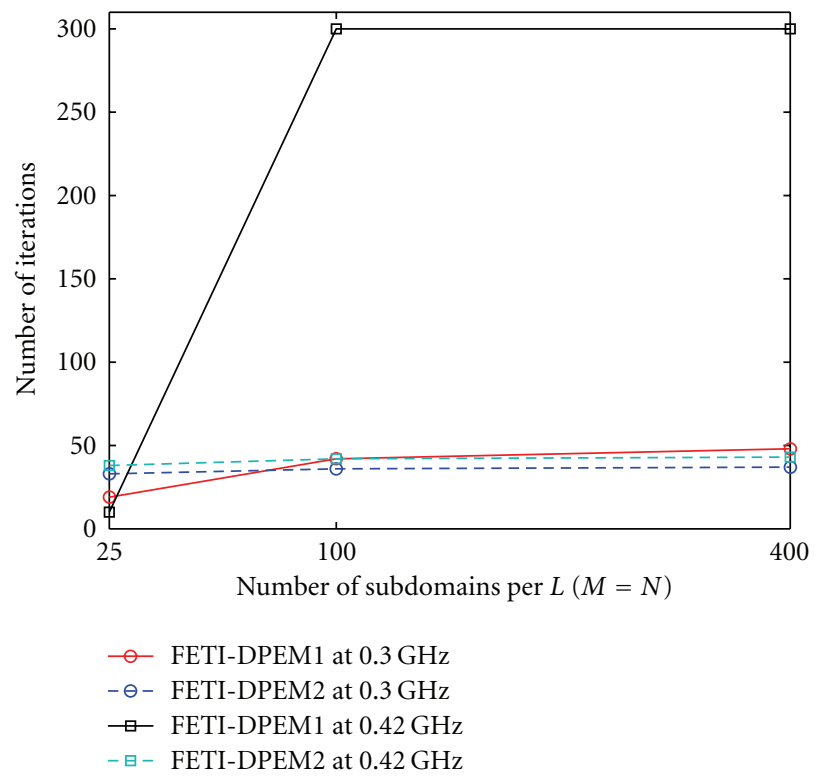

(b) Iteration number versus number of subdomains.

FIGURE 3: Iteration number required by FETI-DPEM1 and FETI-DPEM2.

To demonstrate the efficiency and capability of the two FETI-DPEM methods, the following numerical experiments are performed on a dielectric brick. The absorbing boundary is placed $0.5 \lambda_{0}$ away from the surface of the brick. The solution domain is divided into $M$ segments in $x$-direction and $N$ segments in $y$-direction as shown in Figure 2(a), which is called 2D-extended decomposition. The solution domain also can be divided into $M, N$, and $L$ segments in $x$, $y$, and $z$ directions, respectively, which is called 3D-extended decomposition.

First, let us investigate the performance of FETI for $2 \mathrm{D}$-extended decomposition. The subdomain is a dielectric cube with side length $0.5 \mathrm{~m}$ having $\varepsilon_{r}=4, u_{r}=1$.
Mesh size is fixed as $0.05 \mathrm{~m}$. We increase the frequency of the incident wave from $0.3 \mathrm{GHz}$ to $0.6 \mathrm{GHz}$. The iteration numbers required by FETI-DPEM1 and FETI-DPEM2 with frequency and number of subdomains are presented in Figures 3(a) and 3(b). As we see, the iteration number required by FETI-DPEM2 almost maintains constant with frequency and number of subdomains, whereas that by FETIDPEM1 increase with frequency and number of subdomain, especially for high frequency, which completely agree with the conclusions in $[6,7]$.

Second, let us investigate the performance of FETI for $3 \mathrm{D}$-extended decomposition. We fix subdomain size as $0.2 \mathrm{~m} \times 0.2 \mathrm{~m} \times 0.2 \mathrm{~m}$ with incident frequency $0.3 \mathrm{GHz}$ and 
TABLE 1: Iteration number versus the number of subdomains.

\begin{tabular}{lccccc}
\hline \multirow{2}{*}{$M, N, L$} & \multirow{2}{*}{ Number of subdomains } & \multicolumn{2}{c}{ FETI-DPEM1 } & \multicolumn{2}{c}{ FETI-DPEM2 } \\
& \multicolumn{2}{c}{ Dual unknowns } & Iteration number & Dual unknowns & Iteration number \\
\hline $3,3,3$ & 27 & 2160 & 37 & 4320 & 40 \\
$5,5,5$ & 125 & 12000 & 359 & 24000 & 69 \\
$10,10,10$ & 1000 & 108000 & $>500$ & 21600 & 127 \\
\hline
\end{tabular}

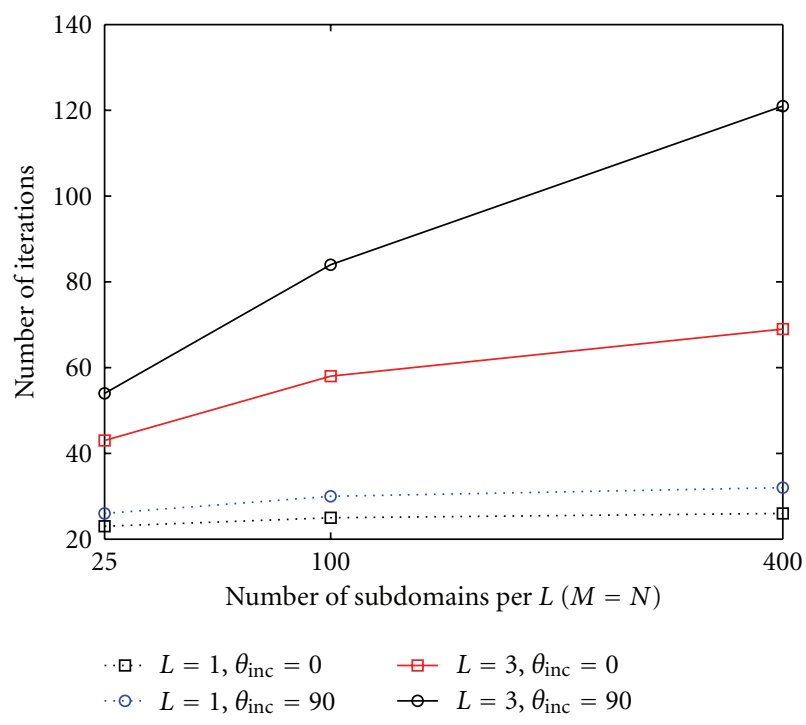

FIGURE 4: Iteration number versus number of subdomains for different $L$.

TABLE 2: Iteration number required by FETI-DPEM2 for dielectric cube with different materials versus $\alpha$.

\begin{tabular}{ccccccc}
\hline$\alpha$ & $j k_{0}$ & $1.5 j k_{0}$ & $2 j k_{0}$ & $2.5 j k_{0}$ & $3 j k_{0}$ & $3.5 j k_{0}$ \\
\hline$\varepsilon_{r}=4$ & 105 & 87 & 84 & 86 & 93 & 103 \\
$\varepsilon_{r}=9$ & 321 & 254 & 233 & 228 & 232 & 250 \\
\hline
\end{tabular}

increase $M, N$, and $L$ at the same time. Table 1 lists number of iterations for FETI-DPEM1 and FETI-DPEM2. It can be seen that the iteration number required by FETI-DPEM2 increases with the number of sub-domains. To further show the differences between different types of domain decomposition, we fix subdomain size as $0.5 \mathrm{~m} \times 0.5 \mathrm{~m} \times 0.5 \mathrm{~m}$ and change $L$ from 1 to 3 . Figure 4 shows the iteration number of the FETI-DPEM2 versus number of subdomains for different $L$ with different incident degree. It can be seen from Figure 4 that, for the 2D-extended domain decomposition, the iteration numbers maintain nearly constant with number of subdomains for different directions of incident wave, but for the 3D-extended domain decomposition, the iteration numbers are not constant again and increase with the number of subdomains. Furthermore, the increase speed of the iteration number with the number of subdomains changes for different incident wave directions. To exclude the possibility due to the increase in number of subdomains, another comparison is provided between $10 \times 10$ with $L=3$



Figure 5: An inhomogeneous dielectric brick with four different materials.

and $30 \times 10$ with $L=1$. The total subdomains are the same, but the numbers of iterations are 58 and 25 , respectively. The latter is nearly the same as those in Figure 4.

Third, we will investigate numerical performance of FETI-DPEM2 versus inhomogeneity. Our numerical experiments are performed on a dielectric cube with $1 \mathrm{~m} \times 1 \mathrm{~m} \times 1 \mathrm{~m}$ at $0.3 \mathrm{GHz}$. We set two cases of the relative dielectric constant $\varepsilon_{r}=4$ and $\varepsilon_{r}=9$, respectively. Mesh size is fixed as $0.05 \mathrm{~m}$ and $0.025 \mathrm{~m}$ for these two cases, respectively. The size of sub-domain is $0.5 \mathrm{~m} \times 0.5 \mathrm{~m} \times 0.5 \mathrm{~m}$, and 64 sub-domains are used. The iteration numbers required by FETI-DPEM2 are 105 and 321 for two cases respectively. It shows that the performance of FETI highly depends on the inhomogeneity of the solution domain. It is worth to point out that in all above computation in FETI-DPEM2, the parameter $\alpha$ in (6) is chosen as $j k_{0}$. In fact, this parameter can be modified to get a better convergence. Table 2 shows that the iteration number required by FETI-DPEM 2 versus $\alpha$. It can be seen from Table 2 that the almost best convergence of FETI-DPEM 2 can be achieved by choosing $\alpha=j k=j \sqrt{\mu_{r} \mu_{0} \varepsilon_{r} \varepsilon_{0}}$, which agrees with that in [12].

To further investigate numerical performance of FETIDPEM2 for inhomogeneity, we perform an experiment for inhomogeneous brick as shown in Figure 5. This brick has four layers, which have different dielectric constants with $\varepsilon_{1}=2, \varepsilon_{2}=3, \varepsilon_{3}=4$, and $\varepsilon_{4}=5$, respectively. The thickness of each layer is $0.5 \mathrm{~m}$. Figure 6 presents the iteration number required by FETI-DPEM 2 versus $\alpha$. It can be obtained from Figure 6 that the optimal performance of FETI-DPEM2 is achieved by choosing $\alpha=j k$, where $k$ can be approximated as average propagation constant of $k=\left(k_{1}+k_{2}+k_{3}+k_{4}\right) / 4=$ $1.84 k_{0}$.

At last, to show the great capability of the FETI-DPEM method, we compute the scattering by a large dielectric brick 
TABLE 3: Computation resources for the large dielectric brick.

\begin{tabular}{|c|c|c|c|c|c|c|}
\hline Material type & $\begin{array}{l}\text { Dual unknowns } \\
(\mathrm{M})\end{array}$ & $\begin{array}{l}\text { Corner unknowns } \\
(\mathrm{M})\end{array}$ & $\begin{array}{l}\text { Total unknowns } \\
(\mathrm{M})\end{array}$ & $\begin{array}{c}\text { Memory } \\
(\mathrm{GB})\end{array}$ & $\begin{array}{l}\text { Iteration } \\
\text { Number }\end{array}$ & $\begin{array}{l}\text { Total CPU time } \\
(\mathrm{Min})\end{array}$ \\
\hline Homogeneous & & & & & 215 & 323 \\
\hline $\begin{array}{l}\text { Inhomogeneous } \\
\text { (Lossless) }\end{array}$ & 9.2 & 0.2 & 41 & 8.2 & 388 & 481 \\
\hline $\begin{array}{l}\text { Inhomogeneous } \\
\text { (Lossy) }\end{array}$ & & & & & 54 & 105 \\
\hline
\end{tabular}



FIGURE 6: Iteration number for the brick versus different $\alpha$.

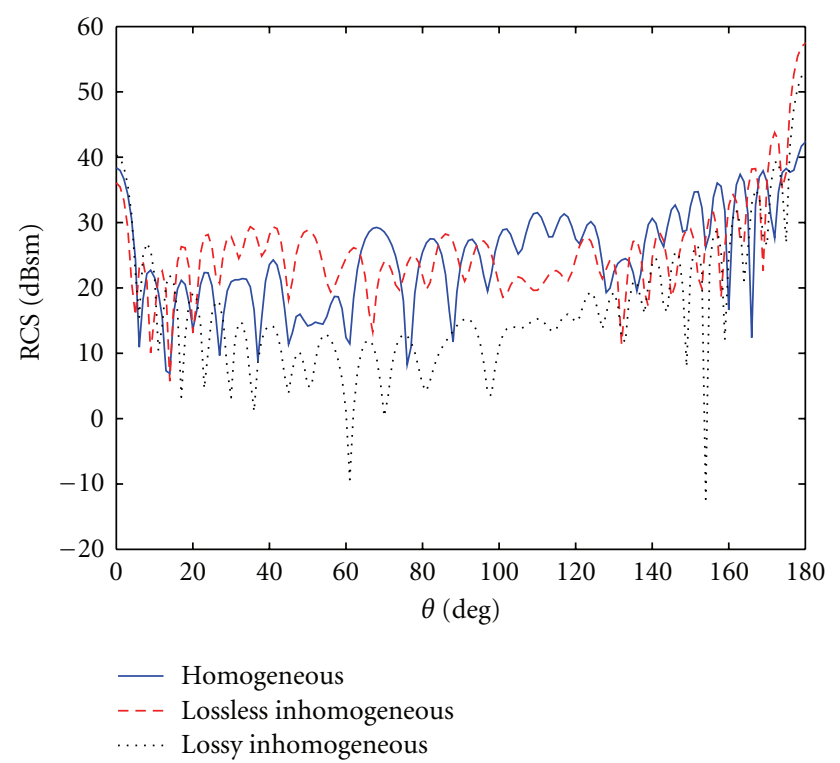

FIgURE 7: Bistatic RCS of the large dielectric brick with $\theta_{\text {inc }}=0^{\circ}$, $\varphi_{\text {inc }}=0^{\circ}$ at $0.3 \mathrm{GHz}$.

up to $10 \mathrm{~m} \times 10 \mathrm{~m} \times 5 \mathrm{~m}$ at $0.3 \mathrm{GHz}$. Three types of material constitutions of the brick are taken into consideration, which are homogeneous, lossless inhomogeneous, and lossy inhomogeneous. For homogeneous case, the brick is filled with material of $\varepsilon_{r}=2$. For lossless inhomogeneous case, the brick is filled with material of $\varepsilon_{r}=2$ and $\varepsilon_{r}=4$ repeatedly along $-z$ direction with thickness of $0.5 \mathrm{~m}$. For lossy inhomogeneous condition, the dielectric constants of material are set to $\varepsilon_{r}=2$ and $\varepsilon_{r}=4-j$, respectively. The bistatic RCSs by these bricks are shown in Figure 7. In these calculations, subdomain size is $0.5 \mathrm{~m} \times 0.5 \mathrm{~m} \times 0.5 \mathrm{~m}$, and the $\mathrm{ABC}$ is placed $0.5 \mathrm{~m}$ away from the brick. Total $22 \times 22 \times 12$ subdomains are used. Computation resources needed in this calculation are listed in Table 3.

\section{Conclusions}

The FETI with the absorbing boundary condition (ABC) is applied to electromagnetic scattering by large inhomogeneous targets in this paper. The convergence speed of FETI-DPEM1 becomes seriously slow with the number of subdomains, especially for high frequency. FETI-DPEM2 is much faster than FETI-DPEM1 with number of subdomains. However, FETI-DPEM2 also cannot maintain the convergence speed with number of sub-domains for the 3D-extended decomposition. Furthermore, the convergence speed of FETI-DPEM2 highly depends on inhomogeneity of targets. Taking inhomogeneity into the coefficient in the Robin transmission condition can improve the convergence speed of FETI-DPEM2.

\section{Acknowledgment}

This work was supported by the NSFC under Grant 10832002.

\section{References}

[1] B. Després, P. Joly, and J. E. Roberts, "A domain decomposition method for the harmonic Maxwell equations," in Iterative Methods in Linear Algebra, pp. 475-484, Elsevier, Amsterdam, The Netherlands, 1992.

[2] Y. J. Li and J. M. Jin, "Implementation of the second-order ABC in the FETI-DPEM method for 3D EM problems," IEEE Transactions on Antennas and Propagation, vol. 56, no. 8, pp. 2765-2769, 2008.

[3] C. Farhat and F. X. Roux, "Method of finite element tearing and interconnecting and its parallel solution algorithm," International Journal for Numerical Methods in Engineering, vol. 32, no. 6, pp. 1205-1227, 1991.

[4] C. T. Wolfe, U. D. Navsariwala, and S. D. Gedney, "An efficient implementation of the finite-element time-domain algorithm on parallel computers using a finite-element tearing and interconnecting algorithm," Microwave and Optical Technology Letters, vol. 16, no. 4, pp. 204-208, 1997. 
[5] C. Farhat, P. Avery, R. Tezaur, and J. Li, "FETI-DPH: a dual-primal domain decomposition method for acoustic scattering," Journal of Computational Acoustics, vol. 13, no. 3, pp. 499-524, 2005.

[6] Y. Li and J. M. Jin, "A vector dual-primal finite element tearing and interconnecting method for solving 3-D large-scale electromagnetic problems," IEEE Transactions on Antennas and Propagation, vol. 54, no. 10, pp. 3000-3009, 2006.

[7] Y. J. Li and J. M. Jin, "A new dual-primal domain decomposition approach for finite element simulation of 3-D large-scale electromagnetic problems," IEEE Transactions on Antennas and Propagation, vol. 55, no. 10, pp. 2803-2810, 2007.

[8] F. X. Roux, F. Magoul'es, L. Series, and Y. Boubendir, "Approximation of optimal interface boundary conditions for two-Lagrange multiplier FETI method," Lecture Notes in Computational Science and Engineering, vol. 40, pp. 283-290, 2005.

[9] M. N. Vouvakis and J. F. Lee, "A fast non-conforming DPFETI domain decomposition method for the solution of large EM problems," in Proceedings of the IEEE Antennas and Propagation Society Symposium, pp. 623-626, June 2004.

[10] J. P. Webb and V. N. Kanellopoulos, "Absorbing boundary conditions for the finite element solution of the vector wave equation," Microwave and Optical Technology Letters, vol. 2, no. 10, pp. 370-372, 1989.

[11] Y. Saad and M. H. Schultz, "GMRES: a generalised minimal residual algorithm for solving nonsymmetric linear systems," SIAM Journal of Scientific and Statistical Computing, vol. 7, pp. 856-869, 1986.

[12] K. Zhao, V. Rawat, S. C. Lee, and J. F. Lee, "A domain decomposition method with nonconformal meshes for finite periodic and semi-periodic structures," IEEE Transactions on Antennas and Propagation, vol. 55, no. 9, pp. 2559-2570, 2007. 



Submit your manuscripts at

http://www.hindawi.com
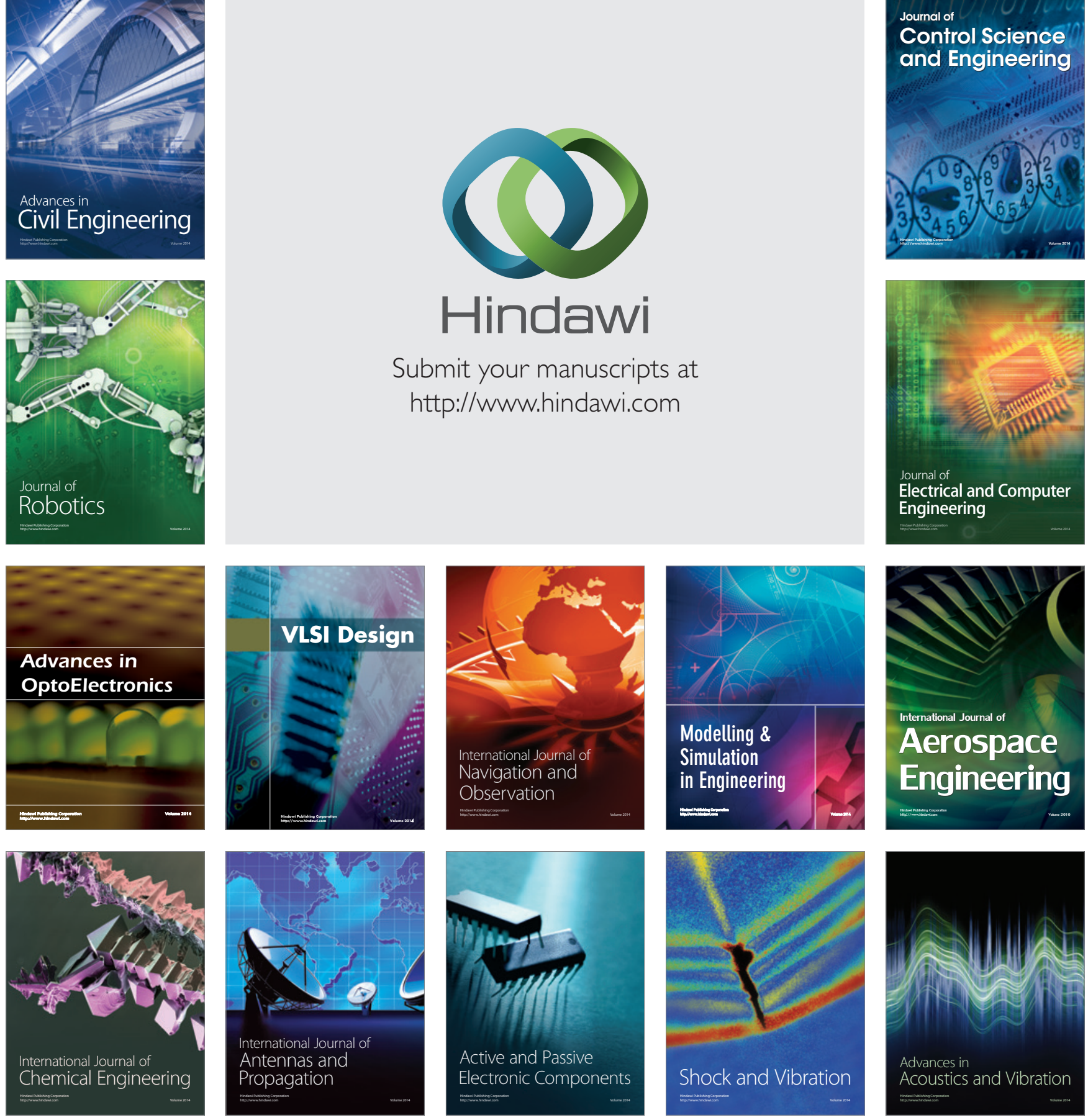